Proceedings

\title{
Ecological Characteristics of Information and Its Scientific Research ${ }^{\dagger}$
}

\author{
Shunpeng Zou ${ }^{1,2}$ and Xiaohui Zou ${ }^{1,2, *}$ \\ 1 China University of Geosciences, Beijing 100083, China; geneculture@icloud.com \\ 2 Searle Research Center, University of California, Berkeley, CA 94720, USA \\ * Correspondence: zouxiaohui@pku.org.cn; Tel.: +86-186-1178-9581; +86-159-1624-1709 \\ + Presented at the IS4SI 2017 Summit DIGITALISATION FOR A SUSTAINABLE SOCIETY, Gothenburg, \\ Sweden, 12-16 June 2017.
}

Published: 8 June 2017

\begin{abstract}
This paper aims to explore the ecological characteristics of information and its scientific research from various aspects. Firstly, it explores formal information including eight basic types, and then explores the essence of information involved the logic of position and order or sequence. Finally, it reveals the ecological characteristics of information ontology related to terminology framework and conceptual system, which is characterized by not only the ambiguity of formal semantics and formal information can be automatically identified and eliminated in the computer-aided information processing environment, but also the ambiguity of the content semantics and content information, can pass through language chessboard and knowledge chess game, with essential information and ontological information, to resolve ambiguity. The result is that the basic law of information and its existence is highlighted, and the social and ecological characteristics of information science research are embodied by macro models (ideas and methods) and micro models (Chinese chessboard and English chessboard). Its significance is information ecology and its supporting methodology, formalization and method system to obtain a new breakthrough, specifically for the classification of information phenomenon and attribution on the determination of its scientific basis, which is conducive to the timely identification and resolution of various ambiguities.
\end{abstract}

Keywords: formal information; essential information; ontological information; information ecology

\section{Introduction}

This paper aims to explore ecological characteristics of information and its scientific research from various aspects.

Grammatical information [1], semantic information [2] and pragmatic information [3] all can be further divided into content information [4] and formal information [5-10]. There is a very rich connotation [11] and extension [12] among them. It is not an exaggeration to call information ecology [13]. So how to recognize or to characterize them is the key to the ecological aspects of information. This paper uses the phenomenon of information [14], the nature or essence of information [15] and the ontology of information [16] as three basic perspectives to understand or to operate the three series of information ecology systematically.

\section{Materials or Knowledge Background}

\subsection{The Prelude of Formal Informatics}

If Tarski's division of object language and meta-language is the beginning of formal informatics, then Turing's calculation or computing of formal information with Shannon's 
Statistical Analysis of formal information is the sign of formal informatics. Furthermore, Chomsky's formal analysis of natural language grammar as formal information and the summary of Feng's formal analysis of natural language, as well as the overall concern of grammatical information, semantic information and pragmatic information by Zhong, and even others need to distinguish the formal information and content information for further concerns, then opened the big screen of formal informatics and content informatics for study.

\subsection{The Prelude of Content Informatics}

It is common thing for people to be mixed the formal information with the content information. If Feigenbaum's analysis and calculation of the content information are the beginning of content informatics within knowledge engineering, then Yixin Zhong's thinking systematically on the grammatical information, the semantic information and the pragmatic information is the content of information science to start. The question now is: how to resolve the ambiguities? That is concerned about and have not yet resolved by Tarski, Carnap, ..., Floridi and others.

\section{Methods}

Firstly, it explores formal information including eight basic types, and then explores the essence of information involved the logic of position and order or sequence. Finally, it reveals the ecological characteristics of information ontology related to terminology framework and conceptual system, which is characterized by not only the ambiguity of formal semantics and formal information can be automatically identified and eliminated in the computer-aided information processing environment, but also the ambiguity of the content semantics and content information, can pass through language chessboard and knowledge chess game, with essential information and ontological information, to resolve ambiguity.

\subsection{The Phenomenon of Information}

Pave the way for the application of the formalized method by using generalized text, which highlights the simple and constructive features of the system of formal information. Due to the distinction between formal information and content information, the information ecology can be significantly simplified. Therefore, study the phenomenon of information in this paper can be simplified into the two standard systems described in generalized text.

As can be seen from Figure 1, one side is data or text the language English processing according to ASCII, namely narrow text formal information processing according to existing standard system; the other side is character /voice the language Chinese processing according to $\mathrm{Z}$ standard system, namely "character/formula/table/picture/voice/image/3D/living" the formal information processing to expand the two standard systems within generalized text.

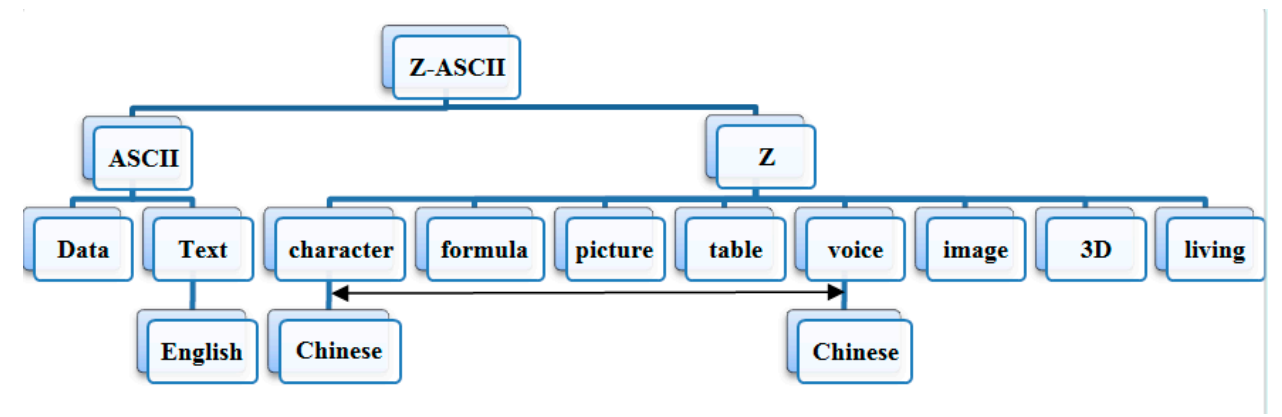

Figure 1. The view of generalized text and its two standard systems Z-ASCII.

It is characterized by the distinction between elements and combinations of generalized text. The two sides together constitute the Z-ASCII standards, which in the chessboards constructed by both numbers and characters embodied in the combination of logic and mathematics for formal information processing system as benchmark reference system that can be fully reflected. 
Thus, it can be said that paving the way through the application of the generalized text for the two formal methods highlights the simple and the constructive features of the formal information system. It can be seen from Figure 1, English and Chinese (including characters and voice) at the bottom, Z-ASCII above, the eight formal systems at one side in the middle that is the object of formal information processing.

To distinguish formal information and content information (to explore essence or nature and class or type) or to consider both sides (to observe phenomenon and example) or both to be treated as a whole (to construct ontology) as three research directions, the formal systems that include "character/formula/table/picture/voice/image/3D/living", in the scope of grammatical information, semantic information, pragmatic information, further, through the phenomenon (includes formal information and content information), reveal the essence.

\subsection{The Essence of Information}

The benchmark reference frame of man-machine brain think tank is obtained by constructing the twin formal tool namely the chessboards with the sequence-position logic and the joint function of generalized bilingual information processing.

The specific chessboards can either be very simple and can also be quite complicated, even super complex. In any case, the characteristics are digital matrix and textual matrix as twins in the sequence-position logic and the generalized bilingual mathematics.

Its role is equivalent to GPS (Global Positioning System) that is for both of GLPS (Language) and GKPS (Knowledge), the difference is that local and global are the same essentially in the logic and the mathematics. In other words, the law followed essentially by logic and mathematics is the same. The basic functions are shown in the following two examples:

Figure 2 (http://kben.koderx.com/article/3/board) shows English formal information processing within mini-chessboards as an example, which is characterized by each basic unit with a specific ID code that is designed to ensure that it is recognized automatically by the machine system while it is invoked every time.

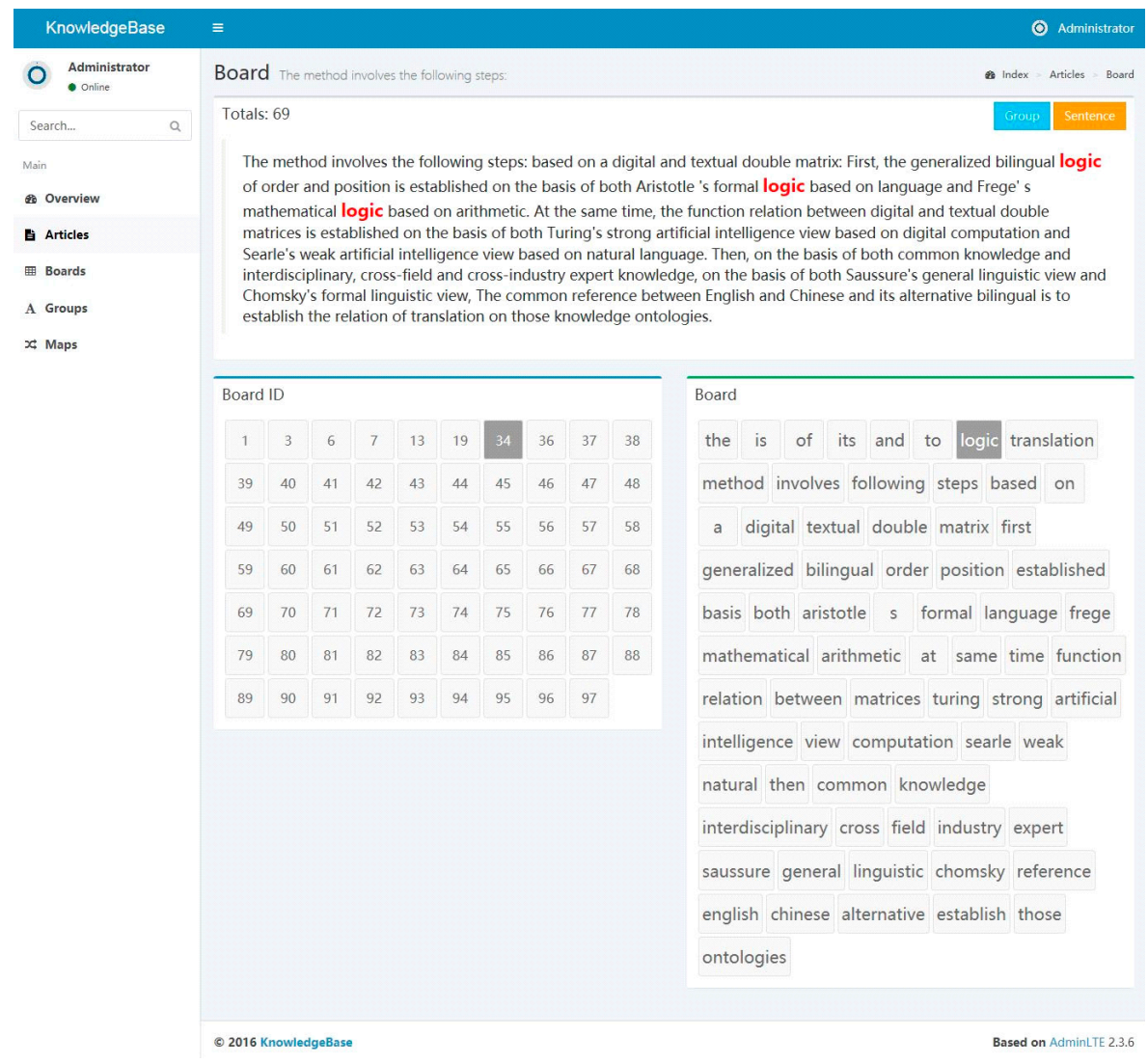

Figure 2. English formal information processing namely micro-chessboards as an example. 
As for the context in which it appears in a specific context, the grammar that follows, specific meaning and specific intention as the richness of the phenomenon of information, the three laws followed by the sequence logic, bilingual mathematics and generalized translation, highlight the essence of information (specific way in the following ontology of information including knowledge ontology or terminology framework).

As can be seen from Figure 3, Chinese formal information processing as an example namely mini-chessboards (http://kb2.sloud.cn/article/51/board), which is characterized by each basic unit with a specific ID code that is designed to ensure that it is recognized by the machine system automatically while it is invoked every time.

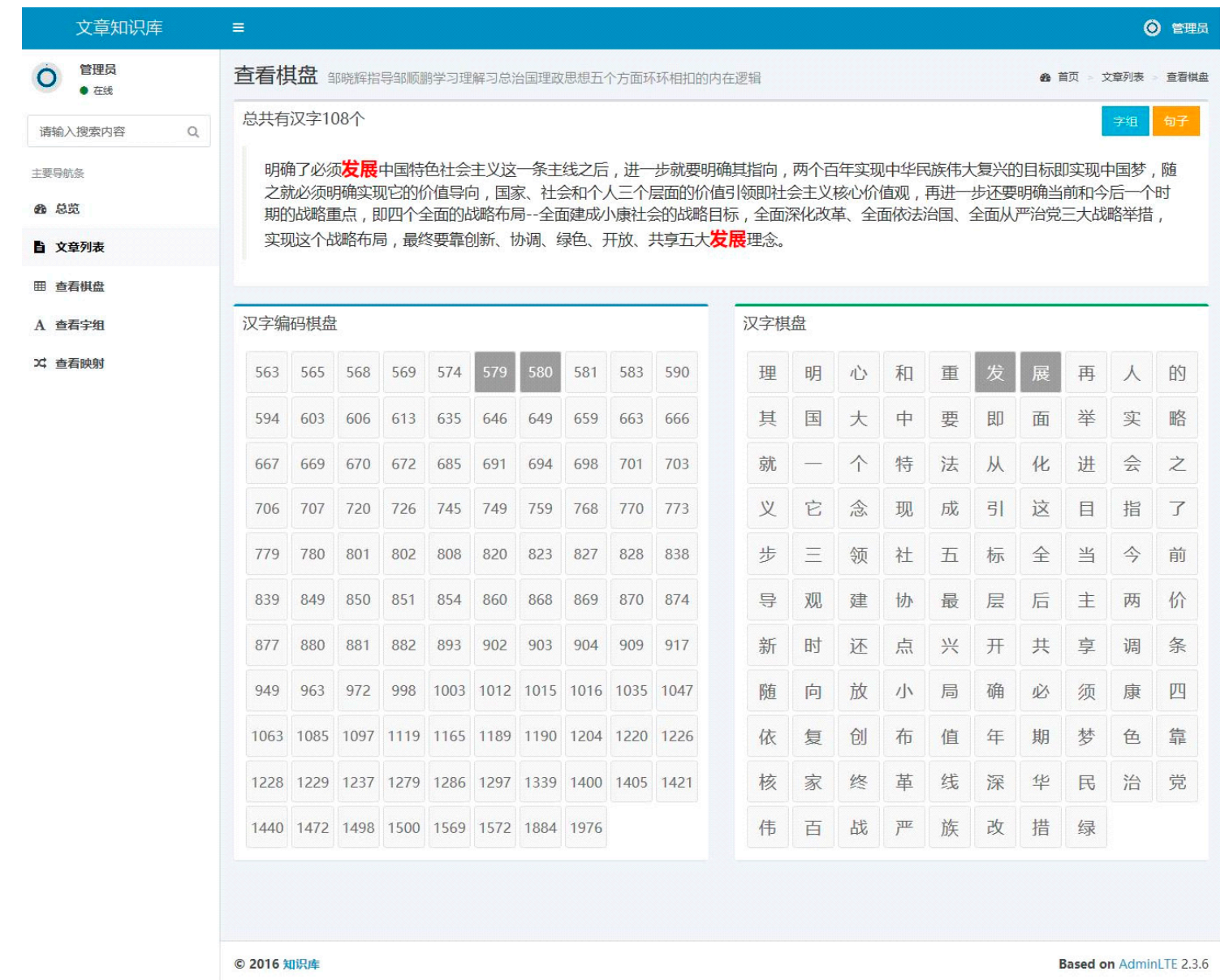

Figure 3. Chinese formal information processing namely mini-chessboards as an example.

As for the context in which it appears in a specific context, the grammar that it follows, specific meaning and specific intention as the richness of the phenomenon of information, can be followed by the sequence logic, bilingual mathematics and generalized translation in the three basic laws, and highlight its essential information (specific way in the follow-up on the ontology of information that is a specific knowledge ontology or terminology frame).

\subsection{The Ontology of Information}

Through the human-computer interaction to confirm the construction of a specific development environment can be formed in the formal expert knowledge system and twin formal expression and even re-used the man- machine twin brains think tank response frame to obtain the equivalent of generalized translation or man-machine coordination to explain knowledge module that constructs knowledge ontology for big production base processing platform.

The construction process and its basic functions can be seen by the following two groups of examples, which can highlight the ontology of information presented accurately, not only to natural 
language understanding (even artificial language understanding), but also to expert knowledge acquisition and its formal expression and reusing in the future (including formal information and content information, co-processing of phenomenon information and essential information), have a special effect (especially in the elimination of misunderstanding or the exclusion of ambiguities, the man-machine twin brains think tank response to reference system, whether it is micro-simple, or giant-complex, construction and reusing are quite completely effective).

It can be seen from Figure 4 as an example of English formal information processing microknowledge chess-menu, which is characterized by word or concept in the locked context, its specific grammar, meaning (semantics) and intention (pragmatic), not only just delineated in form and content of such phenomena information, but also delineated in essential information.

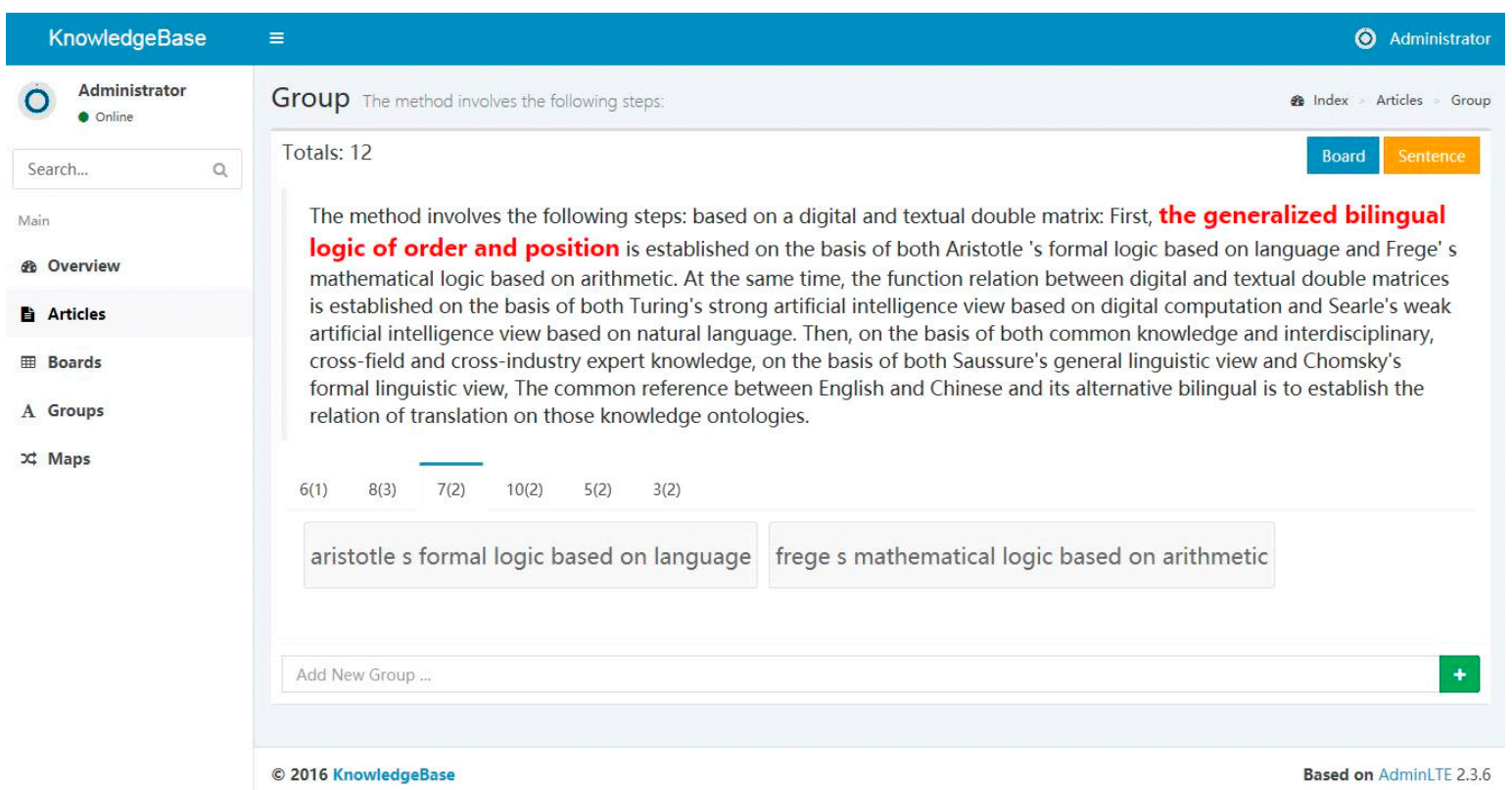

Figure 4. English information processing namely micro-knowledge chess-menu as an example.

In other words, such a set of words or phrases or statements or discourse and even the formal systems have been defined by terminology framework and knowledge ontology. In other words, the computer and the human brain combined with the super twin brains think tank (whether it is micro or giant) for their synergistic understanding and joint expression.

As can be seen from Figure 4, Aristotle's formal logic based on natural language, Frege's mathematical logic based on arithmetic language, Zou's sequence logic based on generalized bilingual (both arithmetic and language) information processing, origin and textual features and interrelations are sufficient to help them in the specific micro and even giant super twin brains think tank to achieve accurate sequencing positioning.

As can be seen from Figure 5 shows Chinese formal information processing namely the microknowledge chess-menu as an example, which is characterized by specific attention to a word or a concept, in the context of locking, its specific grammar, semantics and pragmatics, not only for the phenomenon of information (both form and content) delineated, but also the essence of information delineated.

For example, "Chinese dream" (red) shown in Figure 5, together with the terminology system and the knowledge ontology constructed by the six four-character groups below that, can accurately express the essence of grammar, meaning and intention (to achieve language understanding and knowledge express accurately, to avoid ambiguity or misunderstanding). 


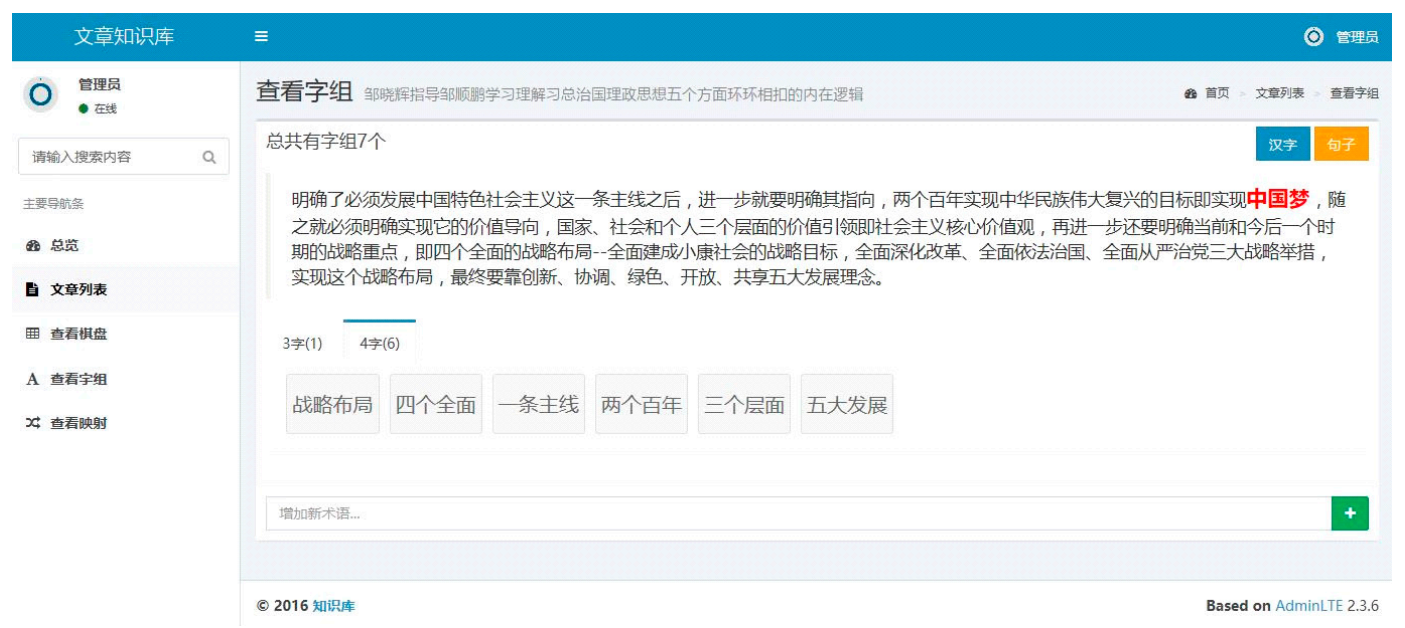

Figure 5. Chinese formal information processing namely micro-knowledge chess-menu as an example.

\section{Results}

This study takes three aspects of phenomenon, essence, ontology as comprehensive information science research approach.

The results reveal the ecological characteristics of information science research and its basic law. Grammar information, meaning information and physical information, can be divided into the phenomenon of information and the nature of information. And that can be further based on mixed sets, logo set, layered set, and single set. Thus, the content information can be distinguished from the formal information. Ambiguous question, here, you can further follow the different basic view (see Figure 6a) and the corresponding basic method (see Figure $6 \mathrm{~b}$ ) to make specific choice.

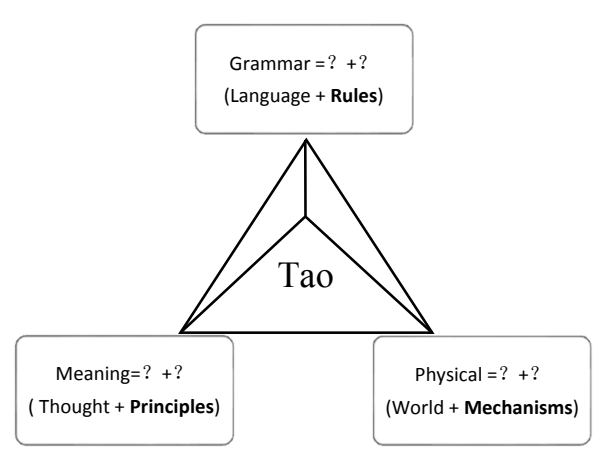

(a)

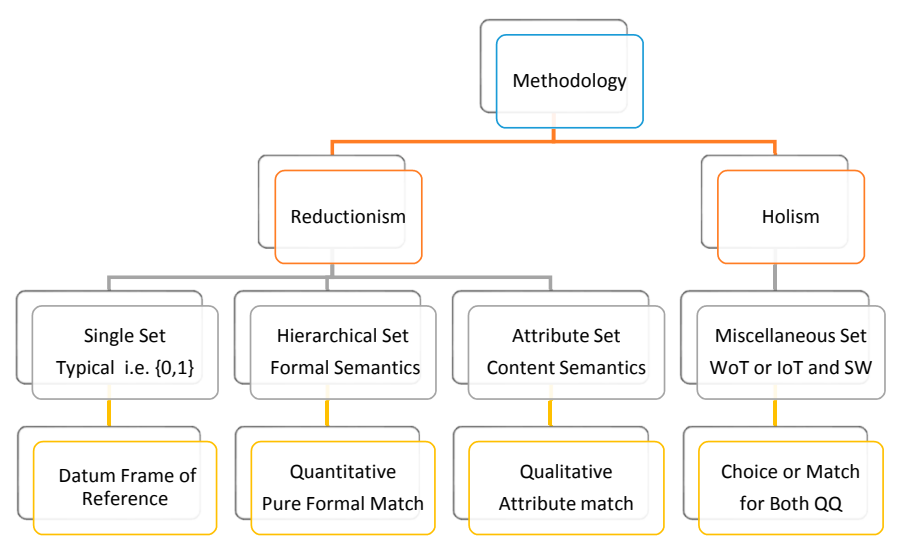

(b)

Figure 6 Basic models of ambiguity analysis: (a) model of basic views; (b) model of basic methods.

The result is that the basic law of information and its existence is highlighted, and the social and ecological characteristics of information science research are embodied by macro models (ideas and methods) and micro models (Chinese chessboard and English chessboard).

\section{Discussion}

In the mathematical theory of communication by Shannon in formal information using statistical analysis method, Wiener's perspective of control with the information feedback but without thinking of mater and energy, can distinguish the three points of view, in fact, pointed out later in the future. The three basic directions of information science research, namely, formal information and content information to treat (explore its essence and class), contact consideration 
(observe phenomenon and example), both distinction and overall grasp or link taken into account (to construct ontology). These are three research directions.

\section{Conclusions}

It is important to discover the basic laws of information science and expression hidden formally in the ecological characteristics of information science research.

As can be seen from Figure 7 shows three equations $\left(I_{d}=n^{2}, I_{d}=I_{k}+I_{u}, I_{k}+I_{u}=m^{2}\right)$ with the twin chess-board $\left(I_{d}=n^{2}, I_{k}+I_{u}=m^{2}\right)$, information identity $\left(I_{d}=I_{k}+I_{u}\right)$, joint function $\left(I_{u}=I_{d}-I_{k}\right)$, based on Z-ASCII system. Further, because of man-machine twin-brains think tank can be either super or miniature, therefore, the human-computer cooperation in the optimal development environment for the knowledge development, either super complex, it can be extremely simple. It can be seen that its meaning is universal. Its significance is information ecology and its supporting methodology, formalization and method system to obtain a new breakthrough, specifically for the classification of information phenomenon and attribution on the determination of its scientific basis, which is conducive to the timely identification and resolution of various ambiguities.

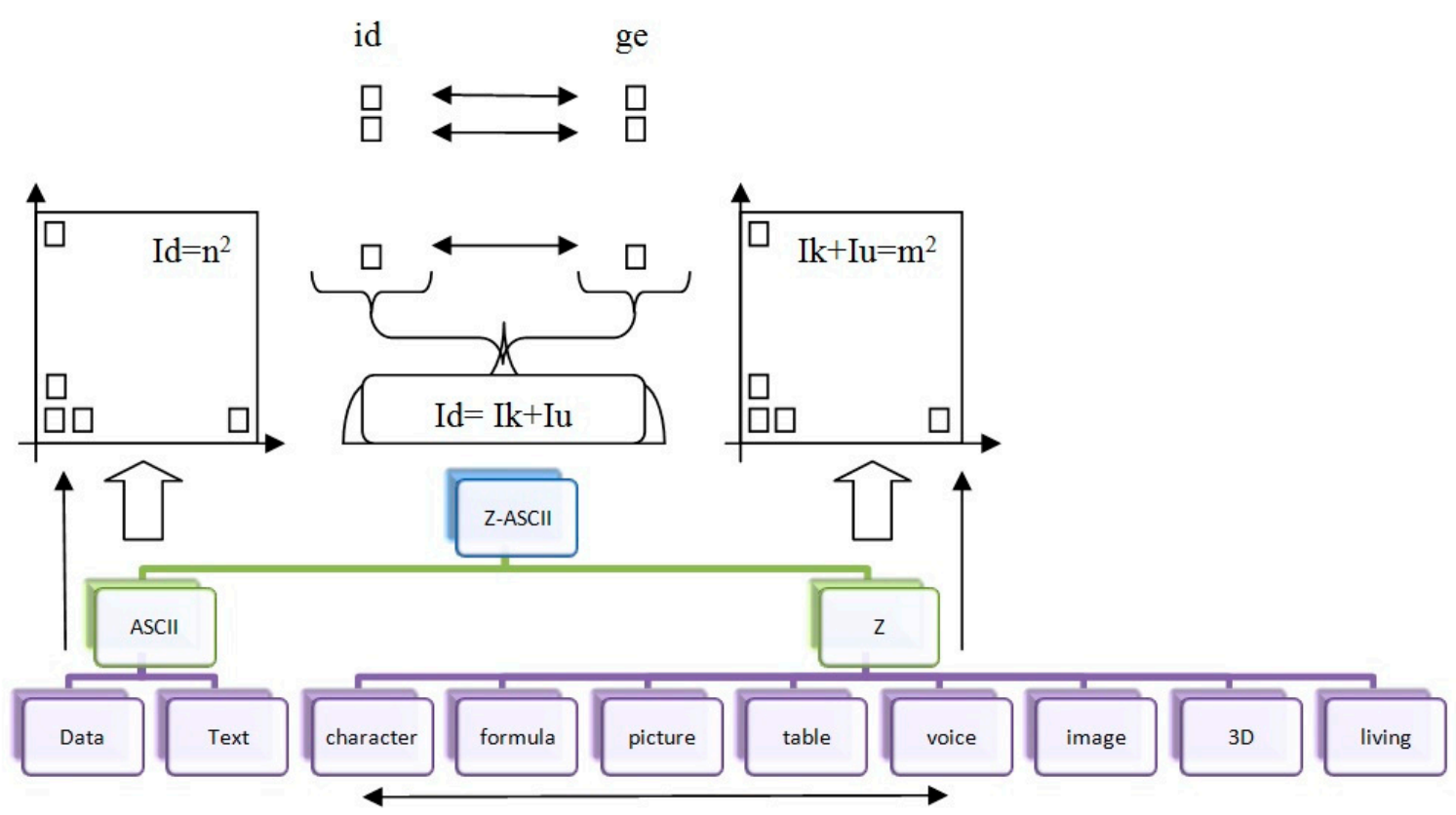

Figure 7 shows three equations with twin chess-board based on Z-ASCII system.

Acknowledgments: All sources of funding of the study have been disclosed.

Author Contributions: Xiaohui Zou conceived and designed the experiments; Xiaohui Zou performed the experiments and analyzed the data; Xiaohui Zou and Shunpeng Zou and Lijun Ke and Riexin Han contributed reagents/materials/analysis tools; Xiaohui Zou wrote the paper.

Conflicts of Interest: The authors declare no conflict of interest. The founding sponsors had no role in the design of the study; in the collection, analyses, or interpretation of data; in the writing of the manuscript, and in the decision to publish the results.

\section{Appendix A}

http://kben.koderx.com/article/3/board http://kben.koderx.com/article/3/group

\section{Appendix B}

http://kb2.sloud.cn/article/51/board http://kb2.sloud.cn/article/51/group 


\section{References}

1. Shannon, C.E. A mathematical theory of communication. Bell Syst. Tech. J. 1948, 27, 379-423.

2. Tarski, A. Surles ensembles définissables de nombres réels. Fundam. Math. 1931, 17, 210-239.

3. Wiener, N. Cybernetics or Control and Communication in the Animal and the Machines; The MIT Press: Cambridge, MA, USA, 1961

4. Zhong, Y. Comprehensive Information Based Methodology for Natural Language Understanding. J. Beijing Univ. Posts Telecommun. 2004, 27. 1-12

5. Turing, A.M. Computing Machinery and Intelligence. Mind 1950, 59, 433-460.

6. Chomsky, N. Syntactic Structures; Mouton Publishers: Paris, France, 1957

7. McCarthy, J.; Minsky, M.; Rochester, N.; Shannon, C.E. A Proposal for the Dartmouth Summer Research Project on Artificial Intelligence, August 31, 1955. AI Mag. 2006, 27, 12-14.

8. Feigenbaum, E.A. The Art of Artificial Intelligence: Themes and Case Studies in Knowledge Engineering. In Proceedings of the 5th International Joint Conference on Artificial Intelligence, Cambridge, MA, USA, 22-25 August 1977; Volume 2.

9. Carnap, R. Introduction to Semantics and Formalization of Logic; Harvard University Press: Cambridge, MA, USA, 1975.

10. Feng, Z. Theory and Method for Formal Analysis of Natural Language by Computer; China University of Science and Technology Press: Hefei, China, 2017. (In Chinese)

11. Zhong, Y. Structuralism? Functionalism? Behhaviorism? or Mechanism? Looking for a better approach to AI. Int. J. Intell. Comput. Cybern. 2008, 1, 325-336.

12. Zou, X. Rational Standard Cooperating Intelligent Model. Lead. Sci. 2005, 149-154. (In Chinese)

13. Zou, X. Fundamental Research of Informatics. In Information Science Inter-Disciplinary Research; Ma, A., Miao, D., Jiang, L., Yan, X., Eds.; 2007; pp. 87-95. (In Chinese)

14. Zhong, Y. Information conversion principle: Information, knowledge, intelligent integration theory. Sci. Bull. 2013, 14, 1300-1306. (In Chinese)

15. Floridi, L. Semantic Conceptions of Information; Stanford Encyclopedia of Philosophy: Stanford, CA, USA, 2014.

16. Searle, J.R. Minds, Brains and Programs. Behav. Brain Sci. 1980, 3, 417-457. 